\title{
Custos Associados à Prestação Informal de Cuidados a Pessoas com Demência
}

\section{Costs of Informal Caregiving in Dementia}

\author{
Cátia Luz PIRES ${ }^{1}$, Natália DUARTE ${ }^{1}$, Constança PAÚL ${ }^{1}$, Oscar RIBEIRO $\bigotimes^{2}$
}

Acta Med Port 2020 Sep;33(9):559-567 - https://doi.org/10.20344/amp.11922

\begin{abstract}
RESUMO
Introdução: Sob a perspetiva dos custos assumidos pelo cuidador informal e pessoa cuidada, este estudo pretende quantificar e valorar a utilização de serviços de saúde e sociais, assim como o tempo dedicado aos cuidados prestados em situações de demência. Material e Métodos: Recolheram-se informações sociodemográficas e de utilização de serviços de 123 díades de cuidados. Os custos foram estimados considerando: Setor Saúde (serviços de saúde), Doente/Família (tempo de cuidados) e Outros (serviços sociais, cuidados continuados, outros privados). A avaliação dos custos do Setor Saúde e Outros Setores considerou a pessoa pagadora; para o Setor Doente/Família aplicou-se o método do bem-substituto. Calculou-se o custo mensal para os utilizadores e o valor para 100 díades.
\end{abstract}

Resultados: O custo total dos cuidados informais foi de $€ 61$ 982,2/mês por 100 díades. O valor do Setor Doente/Família (€44 030,0/ mês) contribui com $71,0 \%$ do custo total; os Outros Setores com 20,8\% (€12 887,4/mês), seguido do Setor da Saúde com 8,2\% (€5064,8/mês).

Discussão: O valor obtido para cada díade (€619,8/mês) representa 77,5\% do seu rendimento mensal (mediana = €800,0; amplitude interquartil $=679,0$ ), o que pode condicionar a continuidade dos cuidados em casa. Em face aos montantes apresentados para esta amostra, estima-se a presença de isenção de taxas moderadoras no Setor Saúde e/ou benefícios na comparticipação das respostas sociais e, como tal, uma estimativa em baixa dos gastos apresentados.

Conclusão: Apesar da complexidade na estimação dos custos associados aos cuidados informais na demência, este artigo ilustra o peso significativo que estes podem alcançar para as famílias.

Palavras-chave: Cuidadores; Custos de Cuidados de Saúde; Custos de Doença Demência

\section{ABSTRACT}

Introduction: From a perspective that considers the costs borne by the informal caregiver and the care-recipient, this study quantifies and values the use of health and social services, and the time dedicated to caregiving in dementia situations.

Material and Methods: Sociodemographic information and use of services was obtained from 123 caregiving dyads. Costs with informal care were evaluated and valued considering the Health Sector (health services), Patient/Family Sector (time of caregiving) and Other Sectors (social services, continuous care, other private services). Costs with health and social services considered the paying person; the Patient/Family Sector was valued through the proxy-good method. The costs were calculated per month and the value for 100 dyads.

Results: The total cost of informal care was $€ 61982.2 /$ month per 100 dyads. The Patient/Family Sector value (€44 030.0/month) contributed with $71.0 \%$ of the total costs, Other Sectors with $20.8 \%$ (€12 887.4/month), and the Health Sector with 8.2\% (€5064.8/month). Discussion: The obtained value per dyad (€619.8/month) represents $77.5 \%$ of their monthly income (median $=€ 800.0$; interquartile range $=679.0$ ), which can limit the decision of continuing the care provision at home. Considering the values presented for this specific sample, it is estimated that the exemption of fees in the Health Sector and/or benefits in social services may contribute to a downward estimate of the costs.

Conclusion: Regardless of the complexity in estimating the costs associated with informal caregiving in dementia, this paper provides some insights on the burden they can represent for the family income.

Keywords: Caregivers; Cost of Illness; Dementia; Health Care Costs

\section{INTRODUÇÃO}

Atualmente estima-se a existência de aproximadamente 50 milhões de pessoas com demência em todo o mundo, projetando-se 82 milhões em 2030 e quase o triplo (152 milhões) em 2050. O número de novos casos de demência em cada ano no mundo é de quase 10 milhões. A repercussão destes números ao nível dos custos globais estima-se em cerca de 700 mil milhões de euros, o que equivale a mais de $1 \%$ da riqueza produzida mundialmente; estes custos incluem gastos com tratamentos médicos, apoio social e ajuda informal (e.g., perda de rendimento/produtividade por parte dos cuidadores). ${ }^{1,2}$ Vários estudos na Europa a 27 estimaram que os custos com os cuidados informais representam mais de $55 \%$ do total de custos associados ao contexto da demência. ${ }^{3,4}$

Os cuidados informais são cuidados regulares a uma pessoa de modo não remunerado (apesar de poderem receber algum tipo de pagamento nominal ou benefício so(ial) $)^{5}$ e desprovido de um vínculo formal prestado por um elemento da rede social do indivíduo, designadamente um familiar, amigo e/ou vizinho. ${ }^{6}$ Em Portugal, apesar de

1. Centro de Investigação em Tecnologias e Serviços de Saúde - CINTESIS. Instituto de Ciências Biomédicas Abel Salazar. Universidade do Porto. Porto. Portugal.

2. Centro de Investigação em Tecnologias e Serviços de Saúde - CINTESIS Pólo Universidade de Aveiro. Departamento de Educação e Psicologia. Universidade de Aveiro. Aveiro. Portugal.

$\triangle$ Autor correspondente: Oscar Ribeiro. oribeiro@ua.pt

Recebido: 11 de fevereiro de 2019 - Aceite: 13 de junho de 2019 | Copyright @ Ordem dos Médicos 2020 
escassearem estudos de base populacional com representatividade nacional, existem alguns dados epidemiológicos recolhidos na região norte do país que dão conta da expressividade numérica da demência. ${ }^{7} \mathrm{O}$ estudo de Santana et $a^{8}$ apresentava um número estimado de 160287 pessoas com mais de 60 anos com demência em 2013, correspondendo a 5,9\% da população nacional acima dos 60 anos de idade. De acordo com o relatório "Health at a Glance 2017" da OCDE, ${ }^{9}$ a estimativa da prevalência de demência para o nosso país rondava as 205000 pessoas e um número correspondente de cuidadores primários e famílias em risco acrescido de sobrecarga física e distress psicológico. Esta realidade terá contribuído para o reconhecimento oficial da necessidade de estruturar um plano nacional de intervenções em demências que considere não só as necessidades dos pacientes como dos seus cuidadores, ${ }^{10}$ assim como para a discussão e proposta da criação do próprio estatuto do cuidador informal.

Entre 2000 e 2013, Portugal registou um aumento de $53 \%$ da capacidade de respostas sociais dirigidas às pessoas idosas, traduzindo-se em 95700 novos lugares, dos quais 52700 são de apoio domiciliário (crescimento de $108 \%) .{ }^{11}$ Estes valores indicam uma importância crescente no apoio a esta população no seu domicílio, sendo esperado que estes cuidados sejam acompanhados por cuidados familiares. A consciencialização da importância dos cuidados informais em termos económicos, porém, assume um destaque relativamente recente no nosso país ${ }^{12}$ e está muitas vezes estimada em termos do valor que seria cobrado por um profissional (e.g., ajudante familiar) em caso de ausência de um cuidador informal $(\mathrm{Cl}) .^{3}$

Assumir o papel de $\mathrm{Cl}$ pressupõe, não raras vezes, deixar de trabalhar ou reduzir o horário de trabalho, mudar de residência, ou alojar a pessoa cuidada (PC) em sua casa. Estas mudanças têm habitualmente grandes implicações no orçamento familiar. Apesar disso, o número de estudos nacionais que procuram compreender o alcance desta problemática a nível individual, familiar e da economia da sociedade é reduzido. ${ }^{13}$ Constrangimentos metodológicos para estimar os custos de prestar cuidados tendem a estar na base das dificuldades na condução desses estudos, particularmente a identificação da melhor forma de analisar o tempo despendido nessa atividade (e.g., apoio pontual em atividades da vida diária versus apoios de supervisão permanente). Na verdade, o cálculo dos custos não se afigura consensual entre os investigadores, nem é fácil de operacionalizar, sobretudo se o $\mathrm{Cl}$ já for responsável por várias tarefas que beneficiam todo o agregado familiar (e.g., as tarefas de cuidado doméstico).

O presente estudo insere-se num projeto de intervenção comunitária de ampla abrangência geográfica (10 municípios da Área Metropolitana do Porto: Espinho, Gondomar, Maia, Paredes, Porto, Póvoa do Varzim, Santo Tirso, Trofa, Vila do Conde, Vila Nova de Gaia) intitulado "Cuidar de Quem Cuida" - capacitação para a intervenção com cuidadores informais de pessoas com demência. ${ }^{14}$ Trata-se de um projeto que procurou, entre outros objetivos, traçar o perfil de utilização de serviços de saúde e de apoio social das díades de cuidados envolvidas. Reconhecendo-se a importância e a premência de contabilizar os custos económicos inerentes à atividade de cuidar, ${ }^{4,12,13,15-18}$ este estudo tem como objetivo ampliar o conhecimento disponível no nosso país nesse âmbito, ao quantificar e valorar os cuidados informais prestados a pessoas com demência residentes na comunidade. Sob a perspetiva dos custos assumidos pela díade CI-PC, foram tidas em conta as despesas com os serviços de saúde, com as respostas sociais, bem como as relativas ao tempo dedicado aos cuidados prestados. Para além disso, o estudo apresenta o perfil sociodemográfico do $\mathrm{Cl}$ e da $\mathrm{PC}$, e o perfil dos serviços utilizados por estas díades de cuidados.

\section{MATERIAL E MÉTODOS \\ População}

Este estudo considera os $\mathrm{Cl}$ de pessoas com demência residentes na comunidade. As organizações envolvidas (municípios, ONG's e serviços de saúde) no projeto "Cuidar de Quem Cuida"19 sinalizaram os participantes. Os técnicos das organizações abordaram diretamente os utentes avaliando o interesse em participar no projeto. Foram incluídos os $\mathrm{Cl}$ que tinham a seu cargo uma pessoa com défices cognitivos compatíveis com um processo demencial (doença de Alzheimer ou outra demência) e residente na comunidade (num dos municípios da Área Metropolitana do Porto). Foram excluídos os $\mathrm{Cl}$ cujas $\mathrm{PC}$ residiam em estruturas residenciais para pessoas idosas, assim como cuidadores formais. Os utentes que aceitaram participar foram posteriormente abordados pela equipa técnica do projeto CQC para confirmação dos critérios de inclusão/exclusão, esclarecimento de dúvidas sobre o projeto e sobre a participação no estudo. O método de seleção adoptado pode condicionar as características da amostra, nomeadamente, pela esperada vulnerabilidade destes participantes, quer ao nível social, como financeiro. Como tal, as opções e padrões de acesso e utilização de serviços podem ser distintos do perfil da população em geral.

De um total de $219 \mathrm{Cl}$ identificados como potenciais beneficiários do projeto "Cuidar de Quem Cuida" incluímos 140 no presente estudo; os restantes 79 não integraram por motivos relacionados principalmente com falta de disponibilidade (e.g., horários laborais incompatíveis com os horários das sessões do programa psicoeducativo). Dos $140 \mathrm{Cl}, 16$ desistiram de participar, relatando falta de disponibilidade e problemas de saúde (do $\mathrm{Cl}$ ou $\mathrm{PC}$ ), e 1 respondeu a um número reduzido de questões e foi excluído da análise. Deste modo, a amostra final considerada foi de $123 \mathrm{Cl}$. O estudo mereceu parecer favorável da Comissão de Ética do ICBAS.UP ( $\left.n^{\circ} 101 / 2015\right)$ e da ARS Norte $\left(n^{\circ}\right.$ $39 / 2015$ ), tendo sido salvaguardadas todas as questões éticas e de proteção de dados subjacentes.

\section{Recolha e análise de dados}

Avaliaram-se todos os $\mathrm{Cl}$ num momento prévio à participação no programa psicoeducativo, por meio de uma 
entrevista estruturada, onde se incluíam informações sociodemográficas do $\mathrm{Cl}$ (sexo, idade, escolaridade, rendimento mensal da díade, grau de parentesco, co-residência com a PC), e da PC (sexo, idade). Técnicos treinados para o efeito avaliaram o grau de deterioração cognitiva das PC através da Escala de Deterioração Global (GDS), para avaliar o grau de severidade da demência da $P C .^{20} \mathrm{O}$ perfil de utilização de serviços de saúde e sociais pela díade foi avaliado através do Questionário sobre a Utilização de Recursos na Demência (RUD), ${ }^{21}$ que contabiliza consultas de urgência, consultas programadas, produtos de incontinência, utilização de respostas sociais formais e número de horas de cuidados informais prestados, e que considera os 30 dias anteriores à data da entrevista. Estimaram-se os custos associados aos cuidados informais segundo a proposta de Drummond et al, ${ }^{22}$ que definiu quatro componentes de avaliação económica (Setor da Saúde, Setor Doente/Família, Outros Setores e Perda de Produtividade), sendo que este estudo considera as três seguintes:

- Setor da Saúde: episódios de urgência hospitalar, de urgência nos cuidados primários, consultas de Medicina Geral e Familiar, e consultas de especialidade hospitalar (i.e., Medicina Interna, Neurologia, Psiquiatria, Ortopedia), tanto para o $\mathrm{Cl}$ como para a PC. Estes serviços foram valorados tendo em conta os preços vigentes na legislação aplicável, relativamente às taxas moderadoras (episódio de urgência hospitalar: $€ 18,0$; episódio de urgência nos cuidados primários e consultas de Medicina Geral e Familiar: $€ 4,5$; consultas de especialidade hospitalar: $€ 7,0) .{ }^{23-25}$ Foram ainda contabilizados os custos com produtos de incontinência utilizados pela PC. Foi realizada uma consulta ao mercado e considerados sete produtos (fraldas), incluindo marca branca, de dois dos principais hipermercados, tendo chegado ao valor médio unitário de $€ 0,56$.

- Setor Doente/Família: custos relativos ao tempo de prestação de cuidados informais. Aqui calculou-se o número de horas de cuidados quer para apoio nas atividades da vida diária básicas (AVDB; e.g., comer, vestir, mobilizar), quer para o apoio nas atividades da vida diária instrumentais (AVDI; e.g., gerir a medicação, tratar da roupa e tarefas domésticas) e, ainda, cuidados de supervisão. Neste setor, aplicou-se o método do bem-substituto (proxy-good), que avalia o tempo do cuidado informal através dos preços do mercado de trabalho da atividade mais próxima (shadow prices). ${ }^{26-28}$ Deste modo, foram consideradas as categorias profissionais que executam as atividades similares a um $\mathrm{Cl}$, mas em ambiente institucional (i.e., estruturas residenciais para pessoas idosas). Assim, para as AVDB e de supervisão considerou-se o ajudante de ação direta (AAD), cujo valor médio da remuneração à data da recolha de dados (2014/16) era de €3,54/hora; e para a valoração das AVDI assumiu-se a média do rendimento do auxiliar de serviços gerais (ASG), no valor de $€ 3,06 /$ hora. $^{29}$
- Outros Setores: custos reportados pelos participantes relativamente aos serviços de apoio social de que são beneficiários, sejam eles serviços de apoio domiciliário (SAD), centro de dia (CD), serviços de acolhimento temporário (SAT) ou unidades de cuidados continuados (UCC). Incluíram-se ainda os custos com o serviço de apoio formal privado (AFP) providenciado por profissionais no domicílio da $\mathrm{PC}$.

A componente da Perda de Produtividade não foi considerada, uma vez que, do total da amostra, apenas $31 \mathrm{Cl}$ (25\%) desempenhavam uma atividade profissional e, destes, apenas três $(2,4 \%)$ referiram reduzir o período de trabalho para cuidar da pessoa com demência.

Os custos dos serviços de saúde e sociais aqui considerados contemplam a perspetiva da pessoa pagadora (díade de cuidados) e não o custo deste para a sociedade ou para uma determinada entidade (e.g., Serviço Nacional de Saúde, Segurança Social). No Setor da Saúde não foram considerados os custos com a medicação, tratamentos, ou aquisição de dispositivos médicos (e.g., cadeira de rodas e camas articuladas) uma vez que o instrumento utilizado na recolha de informação apenas permitiu conhecer o nível de utilização e não os custos com a sua aquisição/utilização.

Realizaram-se análises descritivas (média, desvio padrão, mediana, amplitude interquartil, frequência absoluta e relativa) para todas as variáveis avaliadas, de acordo com a sua natureza. A mediana e a amplitude interquartil foram utilizadas uma vez que as variáveis apresentavam uma distribuição assimétrica. No âmbito de cada componente de avaliação económica, e para cada variável nela incluída, calculou-se o custo mensal apenas para os utilizadores daquele serviço e, à semelhança de outros estudos, o valor para $100 \mathrm{Cl}-\mathrm{PC} .{ }^{28}$ Por último, calculou-se o custo total por setor para a díade $100 \mathrm{Cl}-\mathrm{PC}$. Realizaram-se todas as análises com o apoio do software Statistical Package for the Social Sciences ${ }^{\circledR}$ (SPSS), versão 22.

\section{RESULTADOS \\ Perfil dos participantes}

A análise descritiva dos $\mathrm{Cl}$ que integraram este estudo evidencia que a prestação de cuidados é maioritariamente desempenhada por mulheres ( $n=101 ; 81,5 \%)$. A média de idades dos $\mathrm{Cl}$ é de 57,4 anos ( $\mathrm{dp}=11,4$ anos), com idade mínima de 26 anos e máxima de 82 anos. A maioria dos cuidadores tem entre um a quatro anos de escolaridade (n $=42 ; 37,8 \%$ ), seguindo-se os que têm entre cinco a nove anos $(n=36 ; 32,4 \%)$. Os rendimentos mensais auferidos pelos $\mathrm{Cl}$ são baixos, sendo que $40 \%(n=44)$ recebe até $€ 530$, seguindo-se os cuidadores que não auferem qualquer rendimento $(n=33 ; 30 \%)$. Relativamente ao grau de parentesco, verifica-se que são os filhos que assumem predominantemente a prestação de cuidados ( $n=77 ; 62,1 \%$ ), seguindo-se os cônjuges ( $n=31,25 \%$ ); a maioria dos $\mathrm{Cl}$ pertence ao agregado familiar da PC ( $n=89,71,8 \%)$.

$\mathrm{O}$ perfil das $\mathrm{PC}$ deste estudo mostra que são na maioria do sexo feminino ( $n=87 ; 70,2 \%$ ), com uma média de idades de 82,2 anos ( $d p=7,8$ anos) situada entre um mínimo 


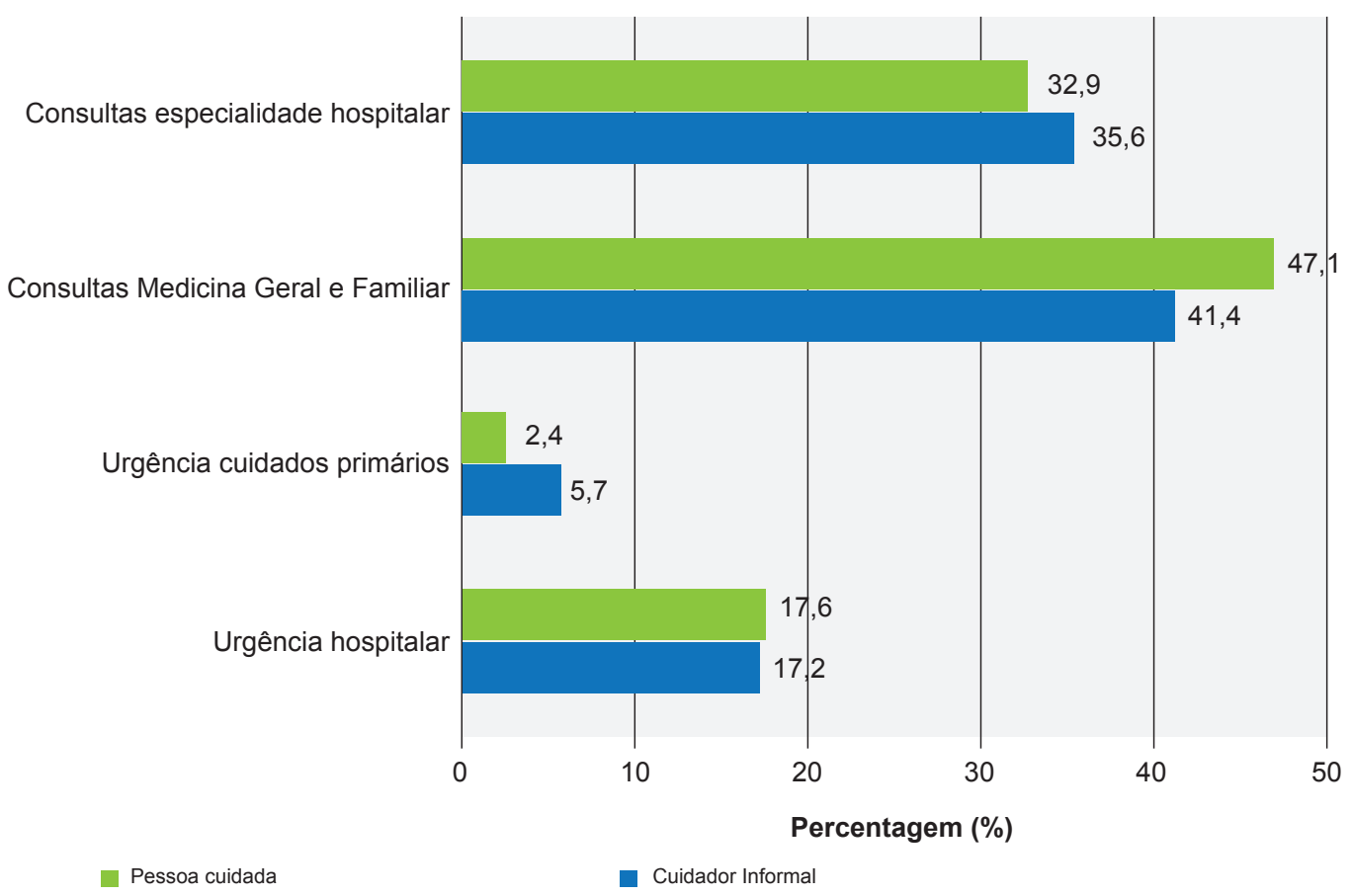

Figura 1 - Caracterização da utilização (\%) mensal de serviços de saúde pela díade CI-PC

de 52 anos e um máximo de 95 anos. A maioria $(n=82$; $66,1 \%$ ) apresenta-se nas fases mais avançadas da demência e, portanto, num grau de dependência muito elevado ou total.

\section{Utilização de serviços de saúde, tempo de prestação de cuidados e respostas sociais}

As Fig.s 1, 2 e 3 apresentam os dados, respetivamente, da utilização de serviços de saúde pelo $\mathrm{Cl}$ e pela $\mathrm{PC}$, dos serviços sociais pela $\mathrm{PC}$ e do tempo despendido pelos $\mathrm{Cl}$ na prestação de cuidados, relativamente aos 30 dias anteriores ao momento de avaliação.
O serviço de urgência hospitalar foi procurado três vezes mais do que a urgência dos cuidados primários (i.e., SAP ou consulta aberta) no caso dos $\mathrm{Cl}$, e sete vezes mais no caso da PC. As consultas de Medicina Geral e Familiar demonstraram maior procura do que as de especialidade hospitalar (Fig. 1). As consultas de especialidade hospitalar mais utilizadas foram as de Medicina Interna $(n=5 ; 5,7 \%)$ e de Psiquiatria ( $n=5 ; 5,7 \%$ ) para o $\mathrm{Cl}$; e as de Neurologia $(n=16 ; 18,8 \%)$ e as de Psiquiatria $(n=7 ; 8,2 \%)$ no caso da PC.

Relativamente à utilização de respostas sociais (Fig. 2), observou-se que o serviço mais requerido era o SAD,

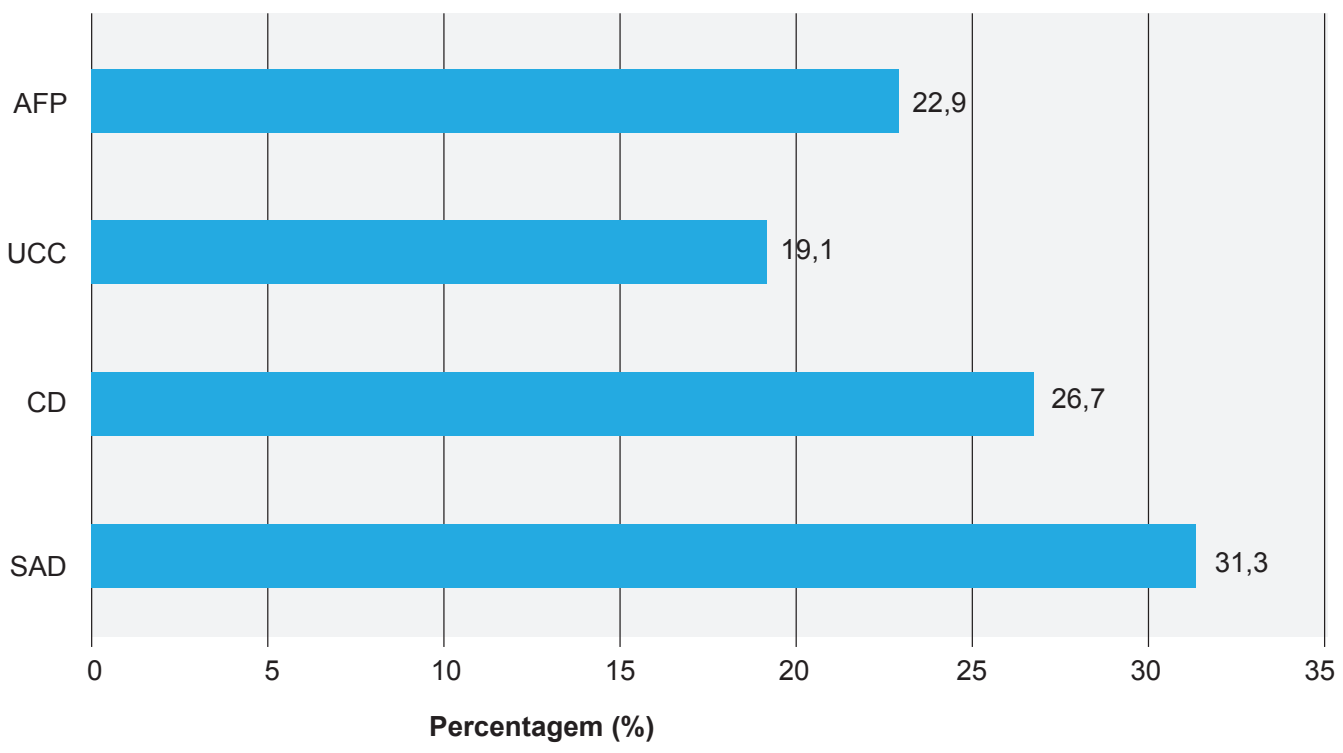

Figura 2 - Caracterização da utilização (\%) mensal de serviços sociais pela PC 


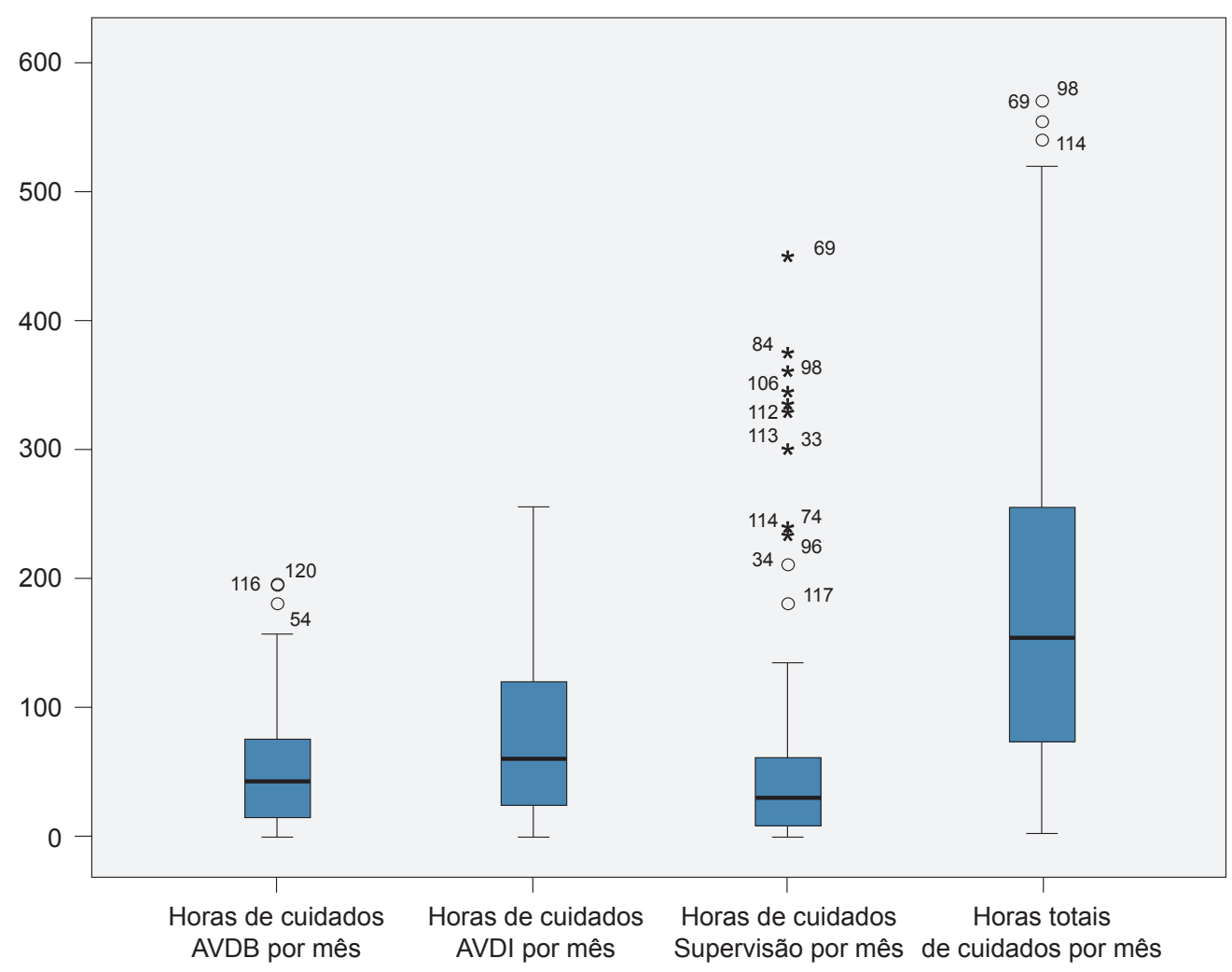

Figura 3 - Caracterização da prestação de cuidados (horas/ mês) por tipologia de cuidados

utilizado por $41(31,3 \%)$ PC, ao qual se segue o $C D(n=35$; $26,7 \%)$. Nenhuma das PC usufruía de SAT.

Quanto ao tempo dedicado à prestação de cuidados (Fig. 3), verificou-se que os $\mathrm{Cl}$ despendem 153,8 horas/ mês $(A I Q=180,0)$, sendo 0 apoio nas AVDI as que exigem um maior dispêndio de tempo (mediana $=60$ horas; $\mathrm{AIQ}$
$=96,4$ ), seguido das AVDB (mediana $=42,5$ horas; $A I Q=$ 61,5 ) e, por último, de supervisão (mediana $=30,0$ horas; $A I Q=52,1)$.

\section{Custos associados aos cuidados informais}

$\mathrm{Na}$ Tabela 1 encontra-se descrito o custo mensal no

Tabela 1 - Custo mensal $(€)$ no Setor da Saúde para o $\mathrm{Cl}$ e PC, aplicando o método do bem substituto

\begin{tabular}{|c|c|c|c|}
\hline & \multicolumn{2}{|c|}{ Utilizadores } & \multirow[t]{2}{*}{ Por $100 \mathrm{Cl}$} \\
\hline & $\mathrm{n}$ & mediana (AIQ) & \\
\hline \multicolumn{4}{|l|}{ Cuidador informal } \\
\hline Urgência hospitalar & 15 & $18,0(18,0)$ & 219,5 \\
\hline Urgência de cuidados primários & 5 & $9,0(4,5)$ & 36,6 \\
\hline Consultas de Medicina Geral e Familiar & 36 & $4,5(0,0)$ & 131,7 \\
\hline Consultas de especialidade hospitalar & 31 & $7,0(7,0)$ & 176,4 \\
\hline \multirow[t]{3}{*}{ Total Setor da Saúde } & & & 564,2 \\
\hline & \multicolumn{2}{|c|}{ Utilizadores } & Por $100 \mathrm{PC}$ \\
\hline & $\mathrm{n}$ & mediana (AIQ) & \\
\hline \multicolumn{4}{|l|}{ Pessoa cuidada } \\
\hline Urgência hospitalar & 15 & $18,0(18,0)$ & 219,5 \\
\hline Urgência de cuidados primários & 2 & $4,5(0,0)$ & 7,3 \\
\hline Consultas de Medicina Geral e Familiar & 40 & $4,5(0,0)$ & 146,3 \\
\hline Consultas de especialidade hospitalar & 28 & $7,0(0,0)$ & 159,3 \\
\hline Fraldas & 87 & $56,1(33,6)$ & 3968,1 \\
\hline Total Setor da Saúde & & & 4500,6 \\
\hline
\end{tabular}


Tabela 2 - Custo mensal $(€)$ do Cl no Setor Doente/ Família, aplicando o método do bem substituto

\begin{tabular}{lc}
\hline & Por $100 \mathrm{Cl}$ \\
\hline Tipologia de apoio & \\
AVDB & 15050,0 \\
AVDI & 18360,0 \\
Supervisão & 10620,0 \\
\hline Total Setor Doente/ Família & 44030,0 \\
\hline
\end{tabular}

Setor da Saúde para o Cl e PC. De um modo geral, destaca-se que o serviço de urgência hospitalar é o que apresenta maior custo ( $€ 219,5 /$ mês por $100 \mathrm{Cl}$ ) e foi utilizado por 15 $\mathrm{Cl}$ no período de 30 dias, com um custo de $€ 18,0 /$ mês por utilizador. As consultas de especialidade foram utilizadas por $31 \mathrm{Cl}$ com um custo de $€ 7,0 /$ mês por utilizador e representam o segundo maior gasto no Setor da Saúde, com um valor de $€ 176,4 /$ mês por $100 \mathrm{Cl}$. Relativamente à $\mathrm{PC}$, o gasto mais representativo (78,3\%) acontece na aquisição de produtos de incontinência com um valor de $€ 56,1 /$ mês, sendo que $70,7 \%$ da amostra revelou necessitar destes produtos. Os restantes $21,7 \%$ dos custos reportam-se aos episódios de urgência e consultas médicas da PC, assumindo o valor de $€ 5,3 /$ mês. O custo mensal total do Setor da Saúde por 100 CI-PC foi de $€ 5064,8 /$ mês.

O custo mensal no Setor Doente/Família para o $\mathrm{Cl}$ apresenta-se na Tabela 2, considerando as categorias de apoio em AVDB, AVDI e Supervisão. O custo total por 100 $\mathrm{Cl}$ foi de $€ 44$ 030,0/mês. Quando analisado o custo por tipos de atividade de cuidado prestado, verifica-se que o maior custo acontece nas AVDI, com €18 360,0/mês, aproximadamente; e com as AVDB, no valor de $€ 15$ 050,0/mês.

Quanto aos Outros Setores (Tabela 3), o custo mensal é representado pelo custo suportado pelas $\mathrm{PC}$, ou respetiva família, quanto ao apoio recebido pelas respostas sociais, apoio formal privado e cuidados continuados. No total por $100 \mathrm{Cl}-\mathrm{PC}$ foram gastos $€ 12887,4 /$ mês, sendo o CD a que representa a maior parcela, correspondente a €4552,5/ mês, e a um valor individual de $€ 160,0 /$ mês considerando os utilizadores deste serviço. Segue-se o SAD, onde os utilizadores gastaram $€ 131,5 /$ mês o que significa que por cada $100 \mathrm{Cl}-\mathrm{PC}$ foram gastos $€ 4383,3 /$ mês. No outro extremo estão os gastos nulos no âmbito das UCC. A resposta de AFP é a que apresenta maior valor individual, na ordem de $€ 180,0 /$ mês.

\section{DISCUSSÃo}

Este estudo permitiu traçar o perfil sociodemográfico e de utilização de serviços de saúde e sociais dos participantes de um projeto de intervenção comunitária destinado a $\mathrm{Cl}$ de pessoas com demência. De um modo geral, a prestação de cuidados revelou ser uma tarefa maioritariamente desempenhada por mulheres de meia-idade, descendentes diretas da PC, com baixo nível de escolaridade e com parcos rendimentos mensais. A PC, na sua maioria mulheres de idade avançada, encontrava-se nas fases mais avançadas da demência e vivia com o $\mathrm{Cl}$. O perfil dos $\mathrm{Cl}$ encontrado no presente estudo é semelhante aos apresentados pelo relatório da Comissão Europeia em $2018^{30}$ e por outros trabalhos científicos, tal como o de Alves el al (2018). ${ }^{31}$ Estes trabalhos mostraram que em Portugal os $\mathrm{Cl}$ são maioritariamente familiares da PC (cônjuges e descendentes), com idades entre os 45 e os 65 anos, residem com a pessoa de quem cuidam, apresentam baixa escolaridade e auferem baixos rendimentos.

As consultas de Medicina Geral e Familiar demonstraram maior procura do que as de especialidade hospitalar, em particular no caso da PC. Não obstante, será necessário atentar ao facto de que foram considerados apenas os 30 dias anteriores ao momento da entrevista para o relato do nível de utilização dos serviços de saúde, pelo que a interpretação destes (e de outros) resultados pode encontrar-se limitada.

Relativamente à procura/ uso de consultas, as PC deste estudo são acompanhadas em contexto hospitalar nas especialidades de Neurologia e de Psiquiatria, existindo igualmente um apoio no âmbito da Medicina Geral e Familiar. Porém, a Psiquiatria surge também como uma das especialidades mais utilizadas pelos $\mathrm{Cl}$. Não ficam claros os contornos da necessidade desta especialidade, mas considerando-se alguns estudos neste âmbito, ${ }^{12,13,18}$ pode dizer-se que provavelmente se deve a níveis de sobrecarga, ansiedade e depressão influenciados pela prestação de cuidados (e.g., sua duração, severidade da doença, grau de apoio psicológico e social recebidos). Quanto ao acesso e uso de serviços de saúde em situação de urgência, verifica-se uma opção preferencial pelos cuidados hospitalares em detrimento dos cuidados primários para ambos os elementos da díade. Não questionando a urgência do estado de saúde, importa destacar que o custo dos cuidados de urgência hospitalar é mais de três vezes superior ao dos cuidados primários e que, apesar de nos últimos anos existir um esforço político de melhoria do acesso, adequação e

Tabela 3 - Custo mensal $(€)$ da PC na Componente Outros Setores, aplicando o método do bem substituto

\begin{tabular}{lccc}
\hline Tipologia de apoio & U & Utilizadores & Por 100 PC \\
\hline SAD & 41 & $131,5(131,0)$ & 4383,3 \\
CD & 35 & $160,0(59,0)$ & 4552,5 \\
UCC & 25 & $0,0(0,0)$ & 0,0 \\
AFP & 27 & $180,0(190,0)$ & 3951,2 \\
Total Outros Setores & & & $\frac{3}{12887,4}$ \\
\hline
\end{tabular}


desempenho dos cuidados primários, ${ }^{32}$ no contexto deste estudo ficou evidente que a escolha recai pela procura dos cuidados hospitalares.

Sendo a demência uma doença degenerativa e progressiva, o cenário de prestação de cuidados encontrado nesta amostra de $\mathrm{Cl}$ recrutados na comunidade, residentes sobretudo com as PC, permite antecipar os constrangimentos decorrentes da sobrecarga social, psicológica e, inevitavelmente, financeira associados à tarefa de cuidar. Efetivamente, os $\mathrm{Cl}$ deste estudo apresentam uma elevada dedicação à prestação de cuidados $(50 \%$ dos $\mathrm{Cl}$ prestam 153,8 horas/mês de cuidados), estando de acordo com os resultados de outros estudos que reportam um maior número de horas de cuidados quando existe coabitação entre o $\mathrm{Cl}$ e a $\mathrm{PC}$, e se está perante um quadro clínico de demência. ${ }^{33}$ No presente estudo, a componente Doente/Família apresentou custos de $€ 44$ 030,0/mês (por $100 \mathrm{Cl}$ ), 71,0\% do custo global, evidenciando a importância da contabilização do tempo de dedicação do $\mathrm{Cl}$ às diferentes tarefas relacionadas com a prestação de cuidados. De salientar que vários estudos na Europa apontam os cuidados informais, diretamente relacionados com o número de horas de prestação de cuidados, como a maior parcela de custos no tratamento e cuidados a pessoas com demência, e que tendem a estar habitualmente ausentes de contabilização nas avaliações económicas em saúde..$^{3,4}$

O Setor da Saúde representa apenas $8,2 \%$ do custo global sendo que destes, $78,3 \%$ reportam-se a custos com produtos de incontinência. Trata-se de um encargo importante, diário, e inteiramente assumido pelo CI-PC. Quanto aos restantes custos com os serviços de saúde, esperava-se que o valor encontrado para o Setor da Saúde fosse mais elevado, indo ao encontro de outros estudos realizados neste contexto, como por exemplo, uma referência a um aumento de $25 \%$ no uso de serviços de saúde pelo facto de se tratar de um cuidado a uma pessoa com doença de Alzheimer. ${ }^{28}$ No entanto, este resultado poderá ser explicado em parte pelo facto de neste estudo não estarem a ser considerados custos com medicação, tratamentos, ou mesmo aquisição de dispositivos médicos ou outros produtos de apoio. Acresce ainda o facto de se tratar de uma amostra que revelou baixos rendimentos, pelo que se espera que muitas destas pessoas estejam isentas de taxas moderadoras e, como tal, reportem gastos diminutos neste setor.

No que respeita ao apoio recebido pelas respostas sociais e cuidados continuados, verifica-se que apesar da maioria das pessoas usufruírem de SAD e/ou CD, é também o AFP que explica os elevados valores deste setor, que representam $20,8 \%$ do custo total dos cuidados informais (€12 887,4/mês por $100 \mathrm{PC}$ ). O valor individual mais elevado é o do AFP ( $€ 180,0 /$ mês), inteiramente assumido pela pessoa. Fica assim evidente a necessidade de um apoio 'extra' que não está a ser satisfeito pelas respostas sociais típicas, nem pelos cuidados continuados, quer seja pela frequência ou periodicidade do cuidado, quer seja pelo tipo de serviço. Infere-se ainda que a disponibilidade das pessoas para pagarem este serviço deixa clara a importância deste para o seu bem-estar.

No que concerne ao custo total dos cuidados informais, sob a perspetiva da díade Cl-PC, objetivo central deste estudo, o seu cálculo com base nas despesas com serviços de saúde, outros serviços sociais e tempo despendido na tarefa de cuidar, revelou ser de €61 982,2/mês para 100 Cl-PC, ou $€ 619,8 /$ mês por díade.

De um modo geral, apesar de entender tratar-se de um valor aparentemente baixo, €619,8/mês representa $77,5 \%$ sobre o rendimento mensal da díade $\mathrm{Cl}-\mathrm{PC}$ (mediana = $€ 800,0 ; A I Q=679,0$ ), o que pode condicionar a decisão da continuidade dos cuidados por parte do $\mathrm{Cl}$ em detrimento da institucionalização da PC. A decisão da família assumir esta responsabilidade encontra-se intrinsecamente relacionada com o equilíbrio entre os ganhos e as perdas inerentes. Se por um lado se identificam como ganhos a valorização, o compromisso do cuidado de alguém que lhe é significativo, por exemplo; por outro, trata-se de abdicar da oportunidade de produzir, do seu rendimento e mais-valias, ou, para o caso de se tratar de pessoas em situação de reforma, de abdicar do tempo de lazer e do desenvolvimento de um conjunto de atividades esperadas, como seja o investimento em relações familiares de cuidado às gerações mais novas.

A grande heterogeneidade inerente aos cuidados informais torna a sua avaliação económica um desafio. Especialmente, importa aqui evidenciar o facto de a recolha dos dados relacionados com os custos ter sido efetuada por autorrelato (em particular, o tempo de dedicação do cuidador às AVDB, AVDI e tarefas de supervisão), podendo ser condicionada por diversos fatores (e.g., enviesamento por execução de atividades conjuntas pelo facto de residirem juntos). ${ }^{5}$ Para além disso, os resultados deste estudo não são (ou não devem ser) generalizáveis, uma vez que se trata de uma amostra sinalizada através de profissionais de respostas sociais ou de saúde, não incluindo os Cl-PC sem ligação a estes organismos. Portanto, um outro perfil de $\mathrm{Cl}$ potencialmente com distintas condições sócio-financeiras poderia evidenciar um outro padrão de acesso e utilização de serviços de saúde e sociais, bem como um outro esforço financeiro inerente à atividade de cuidar (eventualmente, contemplando a contratação de cuidadores formais). Por último, uma vez que os vários estudos realizados neste âmbito adotam distintas metodologias e variáveis, torna-se imprecisa a comparação direta do significado dos custos dos cuidados informais encontrados neste estudo e os reportados na literatura.

\section{CONCLUSÃO}

No âmbito deste estudo, o custo dos cuidados informais calculado com base nas despesas com alguns serviços de saúde, outros serviços sociais e tempo despendido na tarefa para a díade de Cl-PC, revelou ser de $€ 619,8 /$ mês.

A complexidade e heterogeneidade dos cuidados informais não permitem que estes se apresentem com um valor monetário real, mas muito provavelmente subestimado. 
Não obstante, assume-se que este estudo constitui um contributo para o reconhecimento do esforço financeiro que as famílias de cuidadores de pessoas com demência têm no seu dia-a-dia, apelando à consciencialização da sociedade e dos agentes políticos para o significado dos custos presentes na esfera dos cuidados informais. Dada a pertinência atual deste tema, em futuras investigações será importante estender esta metodologia a outros cuidadores.

\section{AGRADECIMENTOS}

A todos os participantes do projeto 'Cuidar de Quem Cuida' (2014 - 2016), à Câmara Municipal de Santa Maria da Feira e ao CASTIIS (Centro de Assistência à Terceira Idade e Infância de Sanguedo), entidade coordenadora do projeto na pessoa da sua diretora, Madalena Malta. Os autores gostariam de agradecer a Susana Oliveira, da Faculdade de Economia da Universidade do Porto, pelo apoio no desenvolvimento do estudo e na análise dos resultados. Um agradecimento também a Ana Filipa Oliveira, bolseira do projeto, pela colaboração na redação do presente documento.

\section{REFERÊNCIAS}

1. World Health Organization. Towards a dementia plan: a WHO guide. Geneva: WHO; 2018.

2. World Health Organization. Global action plan on the public health response to dementia 2017 - 2025. Geneva: WHO; 2017.

3. Wimo A, Jonsson L, Gustavsson A, McDaid D, Ersek K, Georges J, et al. The economic impact of dementia in Europe in 2008 - cost estimates from the Eurocode project. Int J Geriatr Psychiatry. 2011;26:825-32.

4. Gervès C, Chauvin P, Bellager MM. Evaluation of full costs of care for patients with Alzheimer's disease in France: the predominant role of informal care. Health Policy. 2014;116:114-22.

5. Weatherly H, Faria R, Van den Berg B. Valuing informal care for economic evaluation. In: Culyer AJ, editor. Encyclopedia of Health Economics. Volume 3. San Diego: Elsevier; 2014. p. 459-67.

6. Brouwer W, Rutten F, Koopmanschap M. Costing in economic evaluations. In: Drummond M, McGuire A, editores. Economic evaluation in health care: merging theory with practice. New York: Oxford University Press; 2001. p. 68-112.

7. Nunes B, Silva RD, Cruz VT, Roriz JM, Pais J, Silva MC. Prevalence and patterns of cognitive impairment in rural and urban populations from Northern Portugal. BMC Neurol. 2010;10:42.

8. Santana I, Farinha F, Freitas S, Rodrigues V, Carvalho A. The epidemiology of dementia and Alzheimer in Portugal: estimations of prevalence and treatment-costs. Acta Med Port. 2015;28:182-8.

9. OECD. Health at a Glance 2017: OECD Indicators. Paris: OECD Publishing; 2017.

10. Programa Nacional para a Saúde Mental. Portugal - Saúde Mental Em Números - 2015. Lisboa: Direção-Geral da Saúde; 2015.

11. Ministério da Solidariedade, Emprego e Segurança Social. Carta Social - Rede de Serviços e Equipamentos 2013. Lisboa: MSESS; 2013.

12. Caravau H, Barbosa C, Brandão D, Ribeiro O, Martín I. Custos económicos de um programa psicoeducativo. Actas Gerontol. 2013;1:111.

13. Pires CL. Avaliação económica dos cuidados informais à população idosa da região norte de Portugal. Mestrado em Gestão e Economia de Serviços de Saúde. Porto: Faculdade de Economia - Universidade do Porto; 2015.

14. Paúl C, Teixeira L, Duarte N, Pires CL, Ribeiro O. Effects of a community intervention program for dementia on mental health: the importance of secondary caregivers in promoting positive aspects and reducing strain. Community Ment Health J. 2019; 55:296-303.

15. O'Shea E, O'Reilly S. The economic and social cost of dementia in Ireland. Int J Geriatr Psychiatry. 2000;15:208-18.

16. Connolly S, Gillespie P, O'Shea E, Cahill S, Pierce M. Estimating the

\section{PROTEÇÃO DE PESSOAS E ANIMAIS}

Os autores declaram que os procedimentos seguidos estavam de acordo com os regulamentos estabelecidos pelos responsáveis da Comissão de Investigação Clínica e Ética e de acordo com a Declaração de Helsínquia da Associação Médica Mundial.

\section{CONFIDENCIALIDADE DOS DADOS}

Os autores declaram ter seguido os protocolos do seu centro de trabalho acerca da publicação de dados.

\section{CONFLITOS DE INTERESSE}

Os autores declaram não ter conflitos de interesses relacionados com o presente trabalho.

\section{FONTES DE FINANCIAMENTO}

O projeto 'Cuidar de Quem Cuida' foi desenvolvido no âmbito do programa Cidadania Ativa, cujos fundos foram provenientes do Mecanismo Financeiro do Espaço Económico Europeu (EEAGrants), em Portugal geridos pela Fundação Calouste Gulbenkian.

economic and social costs of dementia in Ireland. Dementia. 2014;13:522.

17. Wang $\mathrm{H}, \mathrm{Gao} \mathrm{T}$, Wimo A, Yu X. Caregiver time and cost of home care for Alzheimer's disease: a clinic-based observational study in Beijing, China. Ageing Int. 2010;35:153-65.

18. Van den Berg B, Brouwer W, Van Exel J, Koopmanschap M. Economic valuation of informal care: the contingent valuation method applied to informal caregiving. Health Econ. 2005;14:169-83.

19. Ribeiro O, Duarte N, Paúl C, Malta M, Pires C, Correia L. Projeto Cuidar de Quem Cuida [2014-2016]. Relatório de Avaliação Final do Projeto. Porto: UNIFAI/ICBAS.UP; 2016.

20. Leitão OR, Nina A, Monteiro I. Escala de deterioração global. In: Grupo de Estudos de Envelhecimento Cerebral e Demência, editores. Escalas e testes na demência. Porto: GEECD; 2007. p.10-3.

21. Wimo A, Wetterholm AL, Mastey $V$, Winblad B. Evaluation of the resource utilization and caregiver time in anti-dementia drug trials: a quantitative battery. In: Wimo A, Karlsson G, Jonsson B, Winblad B, editors. The health economics of dementia. London: Wiley; 1998. p.465-99.

22. Drummond MF, Sculpher MJ, Claxton K, Stoddart GL, Torrance GW. Methods for the economic evaluation of health care programmes. $4^{\text {th }}$ ed Oxford: Oxford University Press; 2015.

23. Portaria n64-C/2016. Diário da República, I série, № 63 (2016/03/31).

24. Administração Central do Sistema de Saúde, IP - Circular Normativa n8/2016/DPS/ACSS, 31 março. Alteração do regulamento de aplicação de taxas moderadoras. ACSS, Portugal. Portaria n²34/2015. Diário da República, I série, Nº 153 (2016/08/07).

25. Van den Berg B, Brouwer W, van Exel J, Koopmanschap M, van den Bos GA, Rutten F. Economic valuation of informal care: lessons from the application of the opportunity costs and proxy good methods. Soc Sci Med. 2006;62:835-45.

26. Moore M, Zhu C, Clipp E. Informal costs of dementia care: estimates from the National Longitudinal Caregiver Study. J Gerontol B Psychol Sci Soc Sci. 2001;56B:S219-28.

27. Peña-Longobardo LM, Oliva-Moreno J. Economic valuation and determinants of informal care to people with Alzheimer's disease. Eur J Health Econ. 2015;16:507-15.

28. Ministério do Trabalho e da Solidariedade Social. Boletim do Trabalho e Emprego, n³1, Vol. 82, p. 2521-43. Lisboa: MSESS - Gabinete de Estratégia e Planeamento; 2015.

29. European Comission. Informal care in Europe. Exploring formalisation, availability and quality. Brussels: European Comission; 2018.

30. Alves S, Teixeira L, Azevedo MJ, Duarte M, Ribeiro O, Paul C. Unmet needs in primary care of older clients with mental health concerns. Int $J$ 
Qual Health Care. 2018;1-7.

31. Direção-Geral da Saúde. Plano Nacional de Saúde 2012-2016. 3.2. Eixo Estratégico - Equidade e Acesso aos Cuidados de Saúde. Lisboa: DGS; 2013.

32. Pires CL, Teixeira L, Ribeiro O, Oliveira S. Custo dos cuidados informais a pessoas idosas da região norte de Portugal: aplicação do método do bem substituto. Actas Gerontol. 2016;2:1-7.

33. Marešová P, Dolejš J, Kuca K. Call for a uniform strategy of collecting Alzheimer's disease costs: a review and meta-analysis. J Alzheimer Dis. 2018;63:1-12. 